\title{
SOCIEDAD, TÉCNICA Y LIBERTAD: APUNTES PARA UNA HISTORIA DE LA TÉCNICA
}

\author{
Antonio LÓPEZ PELÁEZ \\ Departamento de Sociología II \\ UNED
}

La reflexión sobre la tecnología contemporánea no puede olvidar el carácter histórico de la técnica, y no sólo como fenómeno concreto, sino como forma de interpretación de la realidad. Por ello, la sociedad tecnológica contemporánea genera, desde el patrón tecnológico, una nueva forma de acceder a la realidad, a la libertad y al proceso de construcción del individuo en la sociedad. La reflexión sobre el impacto social de la tecnologia va unida siempre a la reflexión sobre su origen, sobre su esencia o modo de configuración de la realidad, y genera una doble dimensión, pesimista a ultranza o dogmáticamente optimista, que evoluciona hacia una comprensión crítica que se mantiene en un equilibrio difícil entre ambos polos. $Y$ esto porque la tecnología genera mutaciones de primer orden, y nos enfrenta a nuevos retos en todos los campos. Lo que se debate ante los retos tecnológicos, como señala Adam Schaff, entre otros autores, es el proyecto de hombre que vamos a forjar en los próximo años, y que nos enfrenta con los límites del proyecto ilustrado sobre la humanidad. Por eso es importante acercarse a las reflexiones más potentes sobre la conformación de la técnica, y el sentido que esta genera, ya que, como señala A. Gorz reflexionando sobre el trabajo, las señas de identidad, y las formas de autorrealización, sufren una modificación radical que transforma las bases más profundas de la civilización de los dos últimos siglos, y nos sitúa ante retos que cuestionan y a la vez revitalizan viejas aspiraciones sobre la realización del hombre. Pero, ¿cuál es la lógica propia de la

Éndoxa: Series Filos6ficas, $n^{\circ} 7,1996$, UNED, Madrid:

Antonio López Peláez: Sociedad, técnica y libertad:

Apuntes para una historia de la técnica.

pp.279-295. 
tecnología, si la tiene, como producto humano? ¿Es la confirmación del ideal burgués que centra la identidad humana en la acción racional y la producción material, que ya criticaba el joven Schlegel? ¿O más bien pone en cuestión nuestra civilización, y nos ofrece medios para transformar las condiciones de alienación que han hecho posible el consumo masivo y el hombre masa? En este artículo, nos vamos a centrar, de cara a una profundización en la sociedad tecnológica, en las reflexiones de dos autores que han conformado las reflexiones de sociólogos y filósofos sobre la tecnología y su impacto (y procedencia) en la sociedad actual: analizaremos la comprensión de la técnica contemporánea como fenómeno histórico en Ortega y Heidegger.

La comprensión de la técnica como un fenómeno histórico caracteriza la meditación de Ortega y de Heidegger sobre la técnica. Ambos autores muestran el carácter histórico, evolutivo, de los fenómenos técnicos, así como la especificidad de la técnica moderna, que como fenómeno histórico tiene un origen y un posible final. Pero el acuerdo entre Ortega y Heidegger se limita a la comprensión temporal de la técnica, y a la búsqueda del esquema conceptual en virtud del cual surge y se desarrolla (y que rige el mundo forjado por ella): la esencia de la técnica no es nada técnico, pero la meditación sobre esa dimensión pretécnica arroja en ambos autores resultados diametralmente antagónicos ${ }^{1}$. Entre el "programa pre-técnico" inventado por el hombre (y que constituye su esencia), y el "Gestell" como "envío destinal" del Ser, no puede haber una distancia mayor.

Ambos autores discrepan respecto a la situación previa a la técnica moderna y a la técnica en general. Discrepan en los rasgos fundamentales de la evolución de la técnica, discrepan en torno a su rango ontológico y discrepan también al reflexionar sobre la relación de la técnica con el hombre, con la historia de la metafísica y con el Ser. Para Ortega, la técnica es un instrumento, un

\footnotetext{
${ }^{1}$ Cfr. HeIDegger, M., Die Technik und die Kehre, Pfulligen, Neske 1961, p. 6; ORTEGA Gasset, J., Meditación de la técnica, Obras Completas, vol. V., p. 334.
} 
radical ontológico que permite al hombre construir su propio ser, y su propio mundo. Para Heidegger, la esencia de la técnica moderna nos remite al "Gestell" como enviarse del Ser que es el Ser mismo en cuanto el Ser se da en la forma de destino (aunque no se agota en un destinarse concreto). Este "envio" nos pone en la pista de las huellas del "envío" anterior, la "poíesis", que "resuena" en el "poner" propio de todo destinarse del Ser, y que adopta en el "Ge-stell" un peculiar modo. Por eso, la meditación sobre la esencia de la técnica no es sólo una caracterización de los rasgos esenciales de la realización técnica del mundo, señalando la "com-posición" actual de dicho mundo, sino que nos conduce a la consideración de lo esencial en todo destino: la dimensión del "poner", el destinarse como tal, que adopta en cada caso una forma histórica concreta. Al abrirnos al misterio del Ser, de la donación de sentido, del origen de la historia y de lo que mantiene cada historia, por debajo y unido a los rasgos específicos de cada período, nos acercamos al ámbito esencial en el cual se esencia la historia y cada historia: el Misterio del Ser que oculto otorga. La meditación sobre la donación de sentido que hace posible y constituye el horizonte de inteligibilidad en el cual se desenvuelve una época, nos abre y nos prepara a una nueva "donación", a un nuevo inicio, en cuanto nos situamos más allá y más acá de la lógica interna de la "com-posición" del mundo en que vivimos.

En la historia de la técnica, Ortega encuentra al hombre y su programa como punto de referencia para comprender la diversidad de las técnicas y su evolución. De aquí que el análisis de la historicidad de la técnica y de sus transformaciones enlace con sus reflexiones sobre el cambio histórico, el motivo de la constante innovación del hombre, su condición de heredero, el papel de la cultura recibida como punto de partida desde el cual alcanzar el proyecto de uno mismo (que siempre tiene que ser inventado, frente a la repetición mimética del animal), la relación entre ideas y creencias, entre individuo y sociedad, y entre programa pre-técnico y técnica concreta. Sin embargo, para Heidegger la historia de la técnica es la historia de la consumación de un destinarse del Ser 
que necesita al hombre, pero que está gobernado por el Ser. De aquí que la historia contemporánea, en la que se consuma la esencia de la técnica en su realización planetaria, le lleve a reflexionar sobre la historia de la metafísica, que obedece a dicha esencia y en la que a parece, a nivel conceptual, la misma consumación. $Y$, más allá de nuestro presente como tiempo de la consumación, Heidegger se encaminará hacia una meditación sobre el origen de nuestra época, que le lleva hasta el destino anterior, la "poíesis", y que nos abre a un posible destinarse del Ser que el hombre no puede controlar, sino sólo "esperar". Por eso, la labor del pensar es recoger meditativamente las "insinuaciones" que el "decir" esencial de las palabras nos otorga ${ }^{2}$, permaneciendo en el ámbito del Misterio.

El origen histórico de la técnica moderna se sitúa, según Heidegger, en la época griega. En ella convivía la "poíesis" con el surgimiento del "Gestell" como destino del Ser que se eleva sobre el destino anterior. La esencia de la técnica moderna, como "destino", es una decisión del Ser que se envía, y a la que el hombre "co-responde" de una forma ya asignada de antemano por el Ser. Por lo tanto, es decisión del Ser el comienzo de un nuevo envío, aunque no hay que entender "Ser" al modo de un nuevo primer principio o deidad fundante. De aquí que Heidegger llegue a tachar con un aspa este término, para alejar cualquier interpretación que caiga en los esquemas causales propios de la metafísica. El origen de la historia está más allá de la voluntad del hombre concreto, que se encuentra siempre dentro de una época que ya le ha sido asignada de antemano. Precisamente porque el hombre

\footnotetext{
${ }^{2}$ Heidegger plantea la relación entre "insinuación", pensar instrumental y pensar esencial como una "contribución" que nos prepara para recibir la "insinuación": «Para percatamos de una insinuación se requiere que antes hayamos aplicado el oído al ámbito de donde proviene. Recibir una insinuación es cosa rara y difícil; tanto más rara cuanto más sepamos, y tanto más difícil cuanto más queramos saber. Pero hay también preanuncios de las insinuaciones. A las indicaciones que nos dan, reaccionamos con mayor prontitud y facilidad porque podemos contribuir nosotros mismos a preparar su advenimiento durante un trayecto del camino» HeldeGGrR, M., Was heisst Denken?, Tubingen, Max Niemeyer 1961, p. 91.
} 
sólo puede "esperar", el privilegio de nuestro tiempo aparece en toda su magnitud, en cuanto la consumación del "Gestell" nos remite, por gracia del Ser, al ámbito apropiante de la relación "Ser-hombre", al Misterio". La explicación de la historia en términos de voluntad, de esfuerzo y de programa aparece como una explicación propia del tiempo del "Gestell", que no se acerca a la cuestión esencial: a la luz del enviarse del Ser, no cabe un planteamiento en torno a los términos "responsabilidad", "eficacia" - "proyecto", más allá de la realización de la esencia del hombre en el meditar esencial que permanece en la consideración del hombre como aquel que es puesto por el Ser como el que "co-responde", y en cuya meditación sobre el Ser como Misterio que "oculto otorga" se abre la posibilidad de un nuevo enviarse del Ser que de origen a una nueva época.

Heidegger, por lo tanto, analiza la evolución histórica de la técnica moderna en función de la esencia que la gobierna (esencia que se realiza plenamente en la sociedad tecnológica contemporánea), estableciendo el origen de dicha esencia (que no es sólo el origen de la esencia de la técnica moderna, sino de toda nuestra época, y también de la metafísica) en el destinarse gratuito e incontrolable del Ser. El punto de partida fundamental para comprender la historia de la técnica y su esencia no es el hombre, sino el enviarse del Ser, que genera el sentido que gobierna cada época: el horizonte de inteligibilidad que hace posible y dona el sentido, ya le es dado al hombre concreto en cada caso. La relación del hombre con la técnica, con la sociedad y con el sentido que gobierna ambas y desde el cual cada hombre parte hacia sí mismo, debe comprenderse desde la esencia del hombre como el que "co-responde". A la luz del planteamiento heideggeriano sobre la esencia de la técnica, el pensar esencial aparece como la auténtica acción en la que el hombre desarrolla su ser. Y desde este punto de vista Heidegger puede señalar cómo, frente al humanismo existencialista, el pensar del Ser da a luz un nuevo humanismo

${ }^{3}$ Cfr. HeIdegger, M., Identität und Differenz, Pfullingen, Neske 1957, p. 18. 
que se detiene en la dote asignada por el Ser al hombre: el "co-responder" al Ser en el cual el hombre puede habitar como $\mathrm{tal}^{4}$, y que ya nos ha sido asignado por el Ser mismo. Por eso puede decir que «lo gravísimo da que pensar en el sentido primigenio de que nos encomienda al pensar. Este don que lo gravísimo nos dona es la dote propiamente dicha que se oculta en nuestra esencia». 5 . Es precisamente la comprensión del hombre desde su esencia como aquel que es puesto por el Ser como el que "co-responde" al enviarse del Ser, la que lleva a Heidegger a intentar permanecer meditando en dicha esencia, más allá y más acá del envío concreto del Ser en el que estamos ya de antemano, en nuestro caso el "Gestell". El predominio del Ser determina la dimensión esencial de "espera" que tiene todo pensar que permanece en la proximidad del Misterio, ya que, como señala Heidegger, "el arribo del Ser estriba en el destino del Ser", que no depende del hombre. Este meditar en la esencia del hombre a la luz del Ser es un meditar histórico, que parte de la historia para, a través de ella, alcanzar el destino del Ser que la rige y se esencia en ella, forjando su sentido. De aquí la importancia fundamental de la técnica moderna, ya que ella define la sociedad y la historia en que vivimos. Dado que nuestra historia está conformada por la técnica, debemos volvernos a su esencia para poder alcanzar la esencia de nuestra historia. Desde este "pensar esencial" a la luz de la esencia del hombre como el que "co-responde" al Ser debemos comprender las reflexiones sobre el habitar y el bloque cuádruple que Heidegger desarrolla en Bauen Wohnen Denken?

\footnotetext{
4 *(..) porque esto es humanismo: meditar y preocuparse - curarse- de que el hombre sea humano, y no inhumano, esto es, extraño a su esencia*m, HEIDEGGER, M., Brief über den Humanismus, Gesamtausgabe, Frankfurt, Vittorio Klostermann, 1975-continúa, tomo 9. p. 319.

${ }^{5}$ HeIDEGGER, M., Was heisst Denken?, op. cit., p. 86.

6 Heidegger, M., Brief über den Humanismus, op. cit., pp. 330-331.

${ }^{7}$ HeIdegger, M., Bauen Wohnen Denken, en Vorträge und Aufsätze, Pfullingen, Neske 1954, pp. 145 y ss.
} 
Por lo tanto, la perspectiva heideggeriana aborda los fenómenos técnicos en función del desarrollo histórico de una esencia que en la actualidad ha llegado a su consumación. Es decir, plantea el problema de su inteligibilidad a nivel esencial, remitiéndolos al ámbito de inteligibilidad que los hace posibles y les otorga un determinado significado. Lo importante no es el dato o el acto técnico, sino su sentido, su horizonte de inteligibilidad a la luz del Ser. Desde esta perspectiva puede Heidegger señalar, en Identidad y Diferencia, el sentido profundo de la técnica moderna desde la "co-pertenencia" entre Ser y hombre. $Y$ puede señalar el peligro radical que encierra (a la luz del cual crece también la salvación), a la vez que indica cómo el camino del pensar parte del hecho esencial de nuestro tiempo: la técnica moderna. La dependencia de un objeto del ámbito de inteligibilidad en el cual se inscribe se hace patente a través de la disquisición heideggeriana sobre la fabricación y significado de una copa', que nos muestra la distancia que media entre la interpretación moderna de la causalidad, y la causalidad comprendida al modo griego. Desde una comprensión esencial, la historicidad de la técnica moderna nos remite a su origen, en un determinado momento de la historia (producto de un donarse libérrimo del Ser, que funda una época), al desarrollo de la esencia donada en dicho origen, y a su consumación en nuestro tiempo. La reflexión sobre los instrumentos técnicos, la fecha de los descubrimientos científicos o la evolución de la metodología científico-técnica, es siempre una reflexión histórica que permanece dentro de los límites de la comprensión instrumental de la realidad, que sin ser incorrecta dentro del ámbito definido por el "Gestell", no llega a lo esencial.

En relación con el origen, la dinámica y la consumación de la historia, Heidegger señala siempre la prioridad del Ser, y el papel necesario del hombre en cuanto éste es requerido por el Ser para poder enviarse. La historia de Occidente, a la luz del Ser, se reduce

\footnotetext{
${ }^{8}$ HeIDEGGER, M., Identität und Differenz, op. cit., pp. 20-26.

${ }^{9}$ HeIDEGGER, M., Die Technik und die Kehre, op. cit., pp. 8-11.
} 
a los envíos del Ser, "poíesis" y "Gestell". Y la historia propia del tiempo del "Gestell" se independiza de la acción del hombre como "sujeto" (pese a que la comprensión del hombre como "sujeto" caracteriza la autocomprensión que el hombre tiene de si mismo dentro de la época gobernada por el "Gestell"), remitiéndose al Ser: es la historia de la consumación progresiva de una esencia donada por el Ser. Por lo tanto, dado que el fenómeno técnico es un fenómeno histórico que se revela esencial a lo largo de la historia de Occidente, gobernando la actualidad, en dicho fenómeno técnico tiene que alentar y hacerse visible, desde la interrogación realizada por el pensar esencial, la esencia que gobierna nuestra época. Una esencia, el "Gestell", que se hace visible como esencia de la técnica. Al ser la historia de la metafísica otro fenómeno fundamental de la historia de Occidente, en dicha historia se debe revelar también la esencia de nuestra época como "Gestell", tanto en su origen, cuanto en su desarrollo y consumación actual. De ahí los paralelismos entre la historia de la metafísica y la historia de la técnica: son las dos caras de una misma historia gobernada por una misma esencia: el "Gestell"10.

Desde este planteamiento, Heidegger señalará cómo la Ciencia moderna está gobernada por la esencia de la técnica (al igual que todo fenómeno histórico dentro de la época del "Gestell"). La evolución de la esencia de la técnica, que es la esencia de la metafísica, tiene unas fases, a partir de su origen, que se caracterizan por el desarrollo de una comprensión del mundo y del hombre desde el binomio "sujeto-objeto", culminando en la desaparición de ambos elementos en un "fondo" (Bestand) común definido por el "encargar constante"11. En la actualidad nos

${ }^{10}$ Es interesante señalar cómo el pensamiento heideggeriano rompe la separación entre Ciencia y Técnica, por una parte, y la Historia y la Filosofía, por otra, al encontrar una raíz común que determina el mundo común que forjan y del que surgen. La reducción del campo científico a sus propias pautas de interpretación, ajenas a la percepción literaria, filosófica y artística del mundo, aparece como una etapa que queda relegada ante la realización mundial, en el pensamiento y en la acción, de la esencia de Occidente.

"HeIDEgGer, M., Die Technik und die Kehre, op. cit., pp. 14 y ss. 
situamos más allá de la dinámica "sujeto-objeto" (a pesar de la falsa percepción que el hombre moderno tiene de sí mismo como "señor" de la tierra), en la locura del requerir que caracteriza nuestro tiempo, en el cual se realiza plenamente el proyecto propio de la esencia de la técnica: nos encontramos más allá de dicho proyecto, en el espacio abierto por su consumación ${ }^{12}$. Aunque, según Heidegger, el hombre no se reduce nunca a mero 'fondo ${ }^{13}$. Por lo tanto, nuestro autor señala a lo largo de la historia de la técnica ciertos esquemas conceptuales que caracterizan las fases del desarrollo de la esencia de la técnica moderna, y que quedan superados en el momento de su consumación: al hacerse realidad material la esencia que los gobernaba, estamos más allá de ellos como fases del desarrollo de dicha esencia. La ausencia de "novedad" en el nivel esencial (al reducirse el mundo tecnológico a pura repetición de una determinada interpretación), el puro encargar constante, la absoluta referencia de todo a su consumo y agotamiento, sin identidad en sí mismo, produce la curiosa ausencia de futuro en una sociedad que vive para la novedad constante, para el futuro y lo por-venir. Y desde esta "realización" podemos meditar sobre el mundo occidental reducido a pura "voluntad de voluntad", podemos abrimos al carácter no-técnico de la esencia de la técnica y de la configuración de nuestro mundo. La evolución de la técnica se comprende, pues, desde la afirmación de la esencia de la técnica como modo de desvelación del Ser: un modo de desvelación que llega en nuestro tiempo a su consumación en el nivel de la realización material (porque desde la

${ }^{12}$ Las categorias centrales que han regido dicho proyecto, gobemándolo, y proporcionando el horizonte de inteligibilidad que daba sentido al hacer del hombre y a su comprensión del futuro y de su propio destino, pierden su vigencia al realizarse como tales: ya no queda proyecto, no queda nada que conquistar, sólo queda la desnuda tecnificación universal. La acción basada en dichas categorias ya no es fértil: sólo puede esperar su repetición constante, y esto fomenta la sensación de vacío, de nada, y a la vez la conciencia de la imposibilidad de sustraernos a esa ausencia de proyecto propia del hombre contemporáneo. Aqui encuentra Heidegger la explicación para el dogmatismo de la sociedad científico-técnica, y a la vez la falta de esperanza, que lleva por un lado a la renuncia a pensar, y por otro a la entrega al consumo constante.

${ }^{13}$ HeIdegger, M., Die Technik und die Kehre, op. cit., p. 16. 
perspectiva que permanece en el pensar del Ser, un destino sólo se consuma esencialmente en tanto en cuanto se medita sobre él como un "destinarse" del Ser). El preguntar esencial ejercido sobre la técnica moderna y su historia nos revela su origen histórico en un enviarse del Ser, nos revela su evolución como evolución de dicha esencia del Ser desde su origen hasta su consumación actual, y nos revela la presencia de dos grandes "envíos" del Ser que han dado origen a dos épocas distintas, a dos destinos irreductibles mutuamente desde sus presupuestos internos, pero comunes en cuanto "donaciones" del Ser. Dentro de cada época, los actos técnicos reciben un diverso significado en función de la esencia que gobierna la época de que se trate. Por ello, Heidegger señalará cómo en un nuevo destinarse del Ser la técnica no desaparecerá, sino que sufrirá una variación esencial en su significado a la luz de la esencia donada por el Ser que origine el nuevo destino histórico que quizás suceda a la época del "Gestell".

Frente al planteamiento heideggeriano, Ortega desarrolla una comprensión de la técnica, de los actos técnicos, y de su historia, en función de la vida como realidad radical, ofreciéndonos con ello una hipótesis interpretativa distinta desde la que afrontar la historia de la técnica. Analiza la técnica en función de las necesidades de la vida, que dependen de la idea de la vida que tienen los hombres en cada situación histórica. Precisamente porque la realidad humana es histórica, y consiste en encontrarse ante un sistema de facilidades y dificultades en el que el hombre debe desarrollar su vida, la técnica y los actos técnicos tienen su origen en la vida, y responden al programa inventado que el hombre pretende realizar, y que considera como su propia vida, su auténtica naturaleza. De aquí la distinción orteguiana entre supervivencia animal y el "buenvivir" o "bienestar" propio del hombre ${ }^{14}$. En relación con la historia de la técnica y su evolución,

14 *He aquí por qué el animal es atécnico: se contenta con vivir y con lo objetivamente necesario para el simple existir. Desde el punto de vista del simple existir el animal es insuperable y no necesita la técnica. Pero el hombre es hombre porque para él existir significa desde luego y siempre bienestar; por eso es a nativitate técnico creador de lo 
debemos destacar en la obra de Ortega dos aspectos mutuamente relacionados:

En primer lugar, la técnica es consustancial al hombre, ya que la vida del hombre es lo que éste fabrica guiado por su proyecto: lo humano es construcción. El hombre tiene que fabricarse su vida, realizar su propio programa, que es su esencia; esta vida es siempre artificial en relación con el mundo animal, y su "fabricación" es siempre técnica. Y Ortega no se refiere sólo a la transformación del medio que nos rodea en virtud de nuestro programa, hasta llegar a un mundo propiamente humano; se refiere también a la capacidad cada vez mayor de ensimismarse (que es lo mismo que decir capacidad de ser humano en cuanto tal), de ser cada vez más capaz de abandonar la alteración animal, entrando en el mundo humano propiamente dicho, producto de la fantasía, de la capacidad de imaginar y pensar más allá de los requerimientos de la circunstancia exterior. Este proceso de humanización exige "técnica", actos técnicos que fortalezcan nuestra capacidad de ensimismamiento: lo humano es conquista, mezcla de fantasía y esfuerzo, y en este sentido puede Ortega afirmar que hombre y técnica son sinónimos. De aquí que, en El mito del hombre allende la técnica, Ortega suponga el origen del hombre como un largo esfuerzo de un animal enfermo (enfermo de su propia animalidad o alteración) que, de repente, descubre un mundo interior, se ensimisma, y lucha por aumentar su capacidad de ensimismar$\mathrm{se}^{15}$. En conformidad con el planteamiento orteguiano, al hacer técnico como tal no se le puede buscar un origen histórico: técnica y hombre son consustanciales, y desde el momento que hay hombres, hay técnica. El origen histórico de la técnica es el origen histórico del hombre.

superfluo. Hombre, técnica y bienestar son, en última instancia, sinónimos» ORTFGA $Y$ GASSET, J., Meditación de la técnica, op. cit., p. 329.

15 ORTEGA Y GASSET, J., El mito del hombre allende la técnica, Obras Completas, vol. LX, pp. 621-623. 
En segundo lugar, dado que los actos técnicos están siempre en función de la idea de la vida y del programa de ser que el hombre concreto de cada época histórica quiere realizar; y dado que las necesidades de la vida están en función de lo que el hombre cree que es su vida, su "deseo original", Ortega establece un principio hermenéutico para releer la historia y responder a su verdadera esencia, como historia de los diversos intentos del hombre por cumplir el programa inventado que en cada caso desea e identifica con su propia vida. Toda historia, y la historia de la técnica también, debe ser analizada en relación con la idea de la vida y las necesidades de la vida de cada época. La historia de la técnica debe analizar la evolución de las técnicas en función de la idea de la vida a la que responden y que las constituye. Por eso Ortega distinguirá tres grandes periodos en la historia de la técnica occidental: la técnica del azar, la técnica del artesano y la técnica del técnico ${ }^{16}$. La diferencia esencial entre ellas estriba en la idea que el hombre tiene de la técnica en función de su idea de la vida, y cómo la técnica responde a las necesidades que aparecen como tales a la luz de una determinada idea de la vida. Hay que tener en cuenta, además, el carácter esencial de la técnica para que exista el hombre como tal: la técnica es el radical ontológico que hace posible al hombre como tal, y por lo tanto el hombre no "usa" simplemente la técnica, sino que se forja con ella, y es en ella, tanto en su primitivo origen como especie, cuanto en cada época histórica ${ }^{17}$.

\footnotetext{
16 ORTEGa y Gasset, J., Meditación de la técnica, op. cit., pp. 359-373.

${ }^{17}$ La técnica especifica esencial es, de acuerdo con esto, la capacidad de imaginar y de desear, el proyecto de uno mismo, que surge de un determinado contexto y se eleva hacia la propia identidad, individual y colectiva, utilizando los medios a su alcance, e inventado técnicas que le permitan avanzar en dicha realización. Por eso, el riesgo de la técnica modema occidental es "supremo": atenta contra la capacidad de desearse a sí mismo, construyendo un mundo de hombres masa y niños mimados que se definen por patrones colectivos olvidándose de su propia vida, de su necesidad de ser mediante un proyecto propio. En este camino, Ortega no cae en la ingenuidad del mito del progreso indefinido, y se plantea incluso la posibilidad de una humanidad futura que renuncie incluso a la razón. Pero también advierte contra la imposibilidad de lograr el progreso humano y material en ese medio: un mundo de hombres sin proyecto, de masas, acaba eliminando las condiciones
} 
Desde este punto de vista, al enfrentarnos a la historia de la técnica, no debemos centrarnos sólo en la evolución interna de la técnica occidental (evolución que nos lleva hasta la "técnica del técnico" actual, íntimamente ligada con el desarrollo de la Ciencia y el modo de pensar mecanicista, y que se centra en el domino del medio exterior, de los instrumentos y de la metodología para conseguir los objetivos prefijados, culminando en la percepción de la omnipotencia de la técnica moderna). También debemos dirigirnos hacia otras técnicas que se centran en el cultivo del alma, de la interioridad, y que responden a otra idea de la vida y a otras necesidades vitales definidas en función de dicha idea de la vida. En estas técnicas podemos constatar también una evolución histórica, diferente de la ya analizada. Con esta distinción Ortega propone dos grandes grupos de actos técnicos, que dan lugar a diferentes metodologías técnicas: la técnica dirigida a la transformación del mundo exterior, y la técnica dirigida al mundo interior. Sólo con una visión de conjunto que integre ambas, podremos obtener una visión adecuada de lo que es la técnica en función de la vida, y de la misma vida en su doble dimensión: hacer interno, cultivo de la interioridad, y hacer externo, transformación de la circunstancia en función de nuestro programa previo. Por eso propone una comparación entre las técnicas occidentales y orientales, dado que Occidente ha tomado como punto de referencia el conocimiento de la materia, y Oriente ha tomado. como punto de referencia el conocimiento del alma ${ }^{18}$.

El análisis de la técnica y su historia nos enfrenta, por lo.tanto, al desarrollo de la técnica occidental en virtud de una determinada idea de la vida; nos enfrenta a los dos grandes ámbitos de actuaciones técnicas: la materia y el alma; y nos remite a la vida del hombre, mostrándonos su complejidad, su carácter de esfuerzo

de progreso material que facilita su actual modo de vida. Desde la pérdida de uno mismo sólo cabe una posibilidad: el regreso a la barbarie.

18 ORTEGa y GASSET, J., Meditación de la técnica, op. cit., p. 375. 
en virtud de un determinado programa inventado, y su "artificialidad" en confrontación con el mundo animal. Es decir, la técnica aparece como el radical ontológico que, junto a la fantasía, permite y hace posible algo así como la vida humana. Dada la dependencia de toda técnica del programa "pre-técnico", el principio hermenéutico que debe guiar el análisis de la historia de la técnica es la relación entre técnica e idea de la vida. Lo fundamental es comprender el proyecto del hombre de una época concreta, desde el cual "comprende" su relación con la técnica, y "orienta" el desarrollo técnico en una dirección $u$ otra. A la vez, la historia de la técnica nos introduce en la construcción de la esencia del hombre de cada período, y nos permite acceder al nivel esencial en el cual se logra la realización humana: la elaboración y cumplimiento del deseo de ser, del "deseo original", propio de todo hombre y de toda sociedad, y a partir del cual el hombre concreto construye su identidad. Desde la reflexión sobre la historia de la técnica adquiere pleno sentido la afirmación orteguiana sobre la razón vital como razón histórica: se trata de "narrar", de "contar" la historia, señalando la idea de la vida que gobernaba cada época, y adquiriendo así una comprensión adecuada de los sucesos acaecidos y los logros técnicos en función de las necesidades de la vida tal y como se concebian (lo cual nos permite superar la pura enumeración de datos históricos para entrar de lleno en la construcción del hombre y su esencia histórica).

La superación del idealismo y el mito del progreso indefinido en relación con la historia de la técnica, pasa por la vuelta a la vida como realidad radical. Desde la relación entre técnica, programa pre-técnico y necesidades de la vida, Ortega se enfrenta al optimismo histórico y al mito del progreso, planteando la posibilidad de la desaparición no sólo de la técnica occidental, sino de toda técnica, y con ello de la misma humanidad, que es el resultado de un esfuerzo sostenido durante siglos, a través del cual el hombre abandona la alteración animal, y crea su propia identidad y su propio mundo. En relación con cada técnica concreta, es posible que desaparezca si desaparecen las necesida- 
des humanas que propiciaron su surgimiento (y por eso, en aquellos lugares donde nunca se sintieron tales necesidades, nunca se desarrolló algo así como la técnica occidental $)^{19}$. La crítica del progreso indefinido y necesario, en base a la definición de la vida como esfuerzo y construcción del programa inventado que es la esencia auténtica de cada hombre, no lleva a Ortega a abdicar de la técnica moderna, ni a rechazar o absolutizar la Ciencia: por eso plantea serias dificultades la interpretación de Ortega como miembro de la crítica romántica a la tecnología. Nuestro autor ve su desarrollo como algo positivo. El problema estriba en una comprensión inadecuada de la vida, y aquí se revela el papel central de la razón histórica: ésta nos permite una intelección correcta de la vida como realidad radical, a cuya luz debemos organizar y redefinir la labor técnica y sus objetivos. La vida como realidad radical se convierte en instancia crítica por excelencia. A su luz podemos observar cómo una comprensión inadecuada de la vida nos conduce a un proceso de rebarbarización, como el que Ortega cree ver materializarse en su época. Ahora bien, la enfermedad básica de nuestro tiempo, la crisis del "deseo original", no es producto del avance tecnológico, sino que es resultado de una comprensión errónea de la vida, la idealista, que lleva a concebir de forma equívoca la relación entre hombre y técnica, y entre el individuo y su propia vida. Precisamente por ello la idea de la vida como realidad radical tiene para Ortega un valor "terapéutico", en cuanto nos permite una relación adecuada con nosotros mismos, con la colectividad y con la técnica. Una relación que potencie la esencia humana de cada hombre: la capacidad de "desear-se", de imaginar y realizar su propio programa.

El autor de Meditación de la técnica establece, por lo tanto, un principio radical en su análisis de la técnica: la vida como realidad radical, que nos presenta al individuo y al colectivo de individuos

\footnotetext{
${ }^{19}$ Desde este punto de vista, Orzega propone un criterio de interpretación en relación con las crisis históricas y con las crisis técnicas, que afronta los cambios, al igual que anteriormente hemos señalada el principio hermenéutico que propone el autor español para analizar el surgimiento y consolidación de la técnica moderna occidental, y de toda técnica.
} 
como creadores de su vida, de su mundo, de su propia humanidad y de sus necesidades. Esta "creación" no se puede dar nunca por concluida, ya que todo nuevo ser humano tiene que luchar por desear su propio programa, y por realizarlo (aquí radica el origen del cambio histórico y de las relaciones intergeneracionales). Ortega ofrece un nuevo principio radical que permite, según él, superar la problemática generada por la percepción errónea de la vida propia del realismo, del idealismo y del pensar existencial. La oposición, desde este planteamiento, a la teoría heideggeriana de la historia como designio del Ser en la cual el Ser se dona como destino, retrayéndose siempre a la vez que se envía (meditación que nos presenta la esencia de la técnica como un modo de desvelación del Ser, gobernado por el Ser mismo, y que reflexiona sobre el hombre como aquel cuya esencia consiste en "co-responder" al Ser) no puede ser más radical. La reflexión sobre la técnica nos muestra, según Ortega, al hombre como un ser que, extrañado $e$ inseguro en medio de la circunstancia física y de un mundo natural que no es el suyo, quiere construirse su mundo, en el que pueda descansar, estar seguro y ser feliz, y a ello se entrega con empeño y entusiasmo. Aunque, precisamente por el carácter de esfuerzo y construcción, la seguridad y el mundo humano es siempre algo relativo, siempre en riesgo de desaparecer. Es ese riesgo, definido de forma total por la muerte, el que otorga a la vida humana el carácter dramático en el que tánto insiste el "existencialismo". Pero, para Ortega, la aceptación de la vida transforma dicho dramatismo en esfuerzo heróico y deportivo, en "empresa"20. Desde su propio proyecto y desde sus propias urgencias, Ortega percibió los planteamientos heideggerianos en torno a la técnica formulados en Darmstadt como otra "tesis" opuesta a la suya, a la cual se enfrenta pertrechado de diversos argumentos que favorecen su propia teoría. Esta percepción "errónea" de la obra del autor alemán no le permite alcanzar la intención última del planteamiento heideggeriano, aunque es cierto

${ }^{20}$ ORTEGa Y GASSET, J., Sobre la Razón Histórica, Obras Completas, vol. XII, p. 219. 
que gran parte de la obra de Heidegger posterior a la "Kehre" fue publicada con posterioridad a la muerte de Ortega. Heidegger no pretende establecer una tesis ontológica que supere en radicalismo las anteriores tesis propuestas en la historia de la filosofía, sino permanecer en el ámbito del pensar del Ser, más allá de las interpretaciones conceptuales propias de la época del "Gestell". Sin embargo, lo que caracteriza la obra de Ortega en torno a la historia de la técnica es el choque entre el afán de radicalidad, de superación del pasado y de instauración de un nuevo principio que permita una comprensión más adecuada de la realidad (permitiendo con ello que nos alcancemos y deseemos a nosotros mismos), a la vez que se enfrenta con las consecuencias de la reducción del "ser" a invención, a interpretación.

La reflexión de ambos autores, en su contraste mutuo, nos ofrece, por lo tanto, la posibilidad de enfrentarnos a una comprensión de la historia de la técnica como lugar hermenéutico privilegiado para acceder al hombre, a su destino y a su forma de construirse. Desde ellos podemos abordar una nueva percepción de la historia del rasgo esencial de Occidente, la técnica, interpretándola desde su origen esencial, que no es el puro discurso técnico, sino algo pretécnico, aunque en la meditación sobre esa esencia "pre-técnica" de la técnica discrepen Ortega y Heidegger. Ambos pensadores despejan el camino para una reflexión sobre la historia de Occidente que se aleja de los presupuestos cientifistas, y enlaza de lleno con el problema del significado, de la libertad y de las posibilidades de realización del hombre en cada época. La vertebración de su pensamiento en torno a la esencia de la técnica y a su historia nos permite acceder a las claves centrales de su comprensión de toda historia. $Y$ con ello colaboran decisivamente en favor de la libertad previa que debe regir toda narración histórica, más allá del positivismo exacerbado que empapaba su tiempo, y que, a pesar de la obra de autores posteriores, como Kuhn, todavía hoy ejerce cierta influencia en la Historia de la Ciencia y de la Técnica. 